\title{
The Case for Supporting Inpatient Glycemic Control Programs Now: The Evidence and Beyond
}

\author{
Susan S. Braithwaite, MD, FACP, FACE ${ }^{1}$ \\ Michelle Magee, $\mathrm{MD}^{2,3}$ \\ John M. Sharretts, $\mathrm{MD}^{3}$ \\ Jeffrey L. Schnipper, MD, MPH ${ }^{4,5,6}$ \\ Alpesh Amin, мD, MBA ${ }^{7}$ \\ Gregory Maynard, MD, MSc ${ }^{8}$ \\ ${ }^{1}$ Department of Medicine, University of North \\ Carolina-Chapel Hill, Chapel Hill, North Carolina. \\ ${ }^{2}$ Georgetown University School of Medicine and \\ Healthcare Sciences, Washington, DC. \\ ${ }^{3}$ MedStar Diabetes \& Research Institutes, \\ Washington, DC. \\ ${ }^{4}$ BWH Academic Hospitalist Service, Brigham \\ and Women's Hospital, Boston, Massachusetts. \\ ${ }^{5}$ Division of General Medicine, Brigham and \\ Women's Hospital, Boston, Massachusetts. \\ ${ }^{6}$ Department of Medicine, Harvard Medical \\ School, Boston, Massachusetts. \\ ${ }^{7}$ Department of Medicine, Hospitalist Program, \\ University of California Irvine, Irvine, California. \\ ${ }^{8}$ Department of Medicine, Division of Hospital \\ Medicine, University of California San Diego, San \\ Diego, California.
}

M edical centers are faced with multiple competing priorities when deciding how to focus their improvement efforts and meet the ever expanding menu of publicly reported and regulatory issues. In this article we expand on the rationale for supporting inpatient glycemic control programs as a priority that should be moved near the top of the list. We review the evidence for establishing glycemic range targets, and also review the limitations of this evidence, acknowledging, as does the American Diabetes Association (ADA), that in "both the critical care and non-critical care venue, glycemic goals must take into account the individual patient's situation as well as hospital system support for achieving these goals." ${ }^{, 2}$ We emphasize that inpatient glycemic control programs are needed to address a wide variety of quality and safety issues surrounding the care of the inpatient with diabetes and hyperglycemia, and we wish to elevate the dialogue beyond arguments surrounding adoption of one glycemic target versus another. The Society of Hospital Medicine Glycemic Control Task Force members are not in unanimous agreement with the American Association of Clinical Endocrinologists (AACE)/ADA inpatient glycemic targets. However, we do agree on several other important points, which we will expand on in this article:

1. Uncontrolled hyperglycemia and iatrogenic hypoglycemia are common and potentially dangerous situations that are largely preventable with safe and proven methods.

2. The current state of care for our inpatients with hyperglycemia is unacceptably poor on a broad scale, with substandard education, communication, coordination, and treatment issues.

3. Concerted efforts with changes in the design of the process of care are needed to improve this state of affairs.

\section{DIABETES AND HYPERGLYCEMIA ARE VERY COMMON INPATIENT CONDITIONS}

Diabetes mellitus (DM) has reached epidemic proportions in the United States. A reported $9.3 \%$ of adults over 20 years of age have diabetes, representing over 20 million persons. Despite increasing awareness, diabetes remains undiagnosed in approximately $30 \%$ of these persons. ${ }^{3}$ Concurrent with the increasing prevalence of diabetes in the U.S. population from 1980 through 2003, the number of hospital discharges with diabetes as any 
listed diagnosis more than doubled, going from 2.2 to 5.1 million discharges. ${ }^{4}$ Hospital care for patients with diabetes and hyperglycemia poses a significant health economic burden in the United States, representing over 40 billion dollars in annual direct medical expenditures. ${ }^{5}$

Hyperglycemia in the hospital may be due to known diabetes, to previously unrecognized diabetes, to prediabetes, and/or to the stress of surgery or illness. Deterioration in glycemic control in the hospital setting is most commonly associated with one or more factors, including stress-induced release of insulin counterregulatory hormones (catecholamines, cortisol, glucagon, and growth hormone), exogenous administration of high dose glucocorticoids, and suboptimal glycemic management strategies. ${ }^{6-8}$ In a Belgian medical intensive care unit (MICU) randomized controlled trial (RCT) of strict versus conventional glycemic control, mean blood glucose (BG) on admission to the unit in the intention to treat group was $162 \pm 70$ $\mathrm{mg} / \mathrm{dL}(\mathrm{n}=1200),{ }^{9}$ and in this group's RCT of 1548 surgical intensive care unit (SICU) patients, BG > $110 \mathrm{mg} / \mathrm{dL}$ was observed in over $70 \%$ of subjects. ${ }^{10}$ Mean BG of $>145 \mathrm{mg} / \mathrm{dL}$ has been reported in $39 \%{ }^{11}$ and $\mathrm{BG}>200 \mathrm{mg} / \mathrm{dL}$ in anywhere from $11 \%$ to $31 \%$ of intensive care unit (ICU) patients. ${ }^{10,12}$ For general medicine and surgery, 1 study of 2030 patients admitted to a teaching hospital revealed that $26 \%$ of admissions had a known history of DM and $12 \%$ had new hyperglycemia, as evidenced by an admission or in-hospital fasting BG of $126 \mathrm{mg} / \mathrm{dL}$ or more or a random BG of $200 \mathrm{mg} / \mathrm{dL}$ or more on 2 or more determinations. ${ }^{13}$ National and regional estimates on hospital use maintained by the Agency for Healthcare Research and Quality include data concerning diabetes diagnoses alone, without hyperglycemia, and may be displayed by querying its Web site. ${ }^{14}$ In cardiovascular populations almost $70 \%$ of patients having a first myocardial infarction have been reported to have either known DM, previously unrecognized diabetes, or impaired glucose tolerance. ${ }^{15}$

\section{THE EVIDENCE SUPPORTS INPATIENT GLYCEMIC CONTROL Evidence: Physiology}

The pathophysiologic mechanisms through which hyperglycemia is linked to suboptimal outcomes in the hospital are complex and multifactorial.
Although it is beyond the scope of this article to discuss these mechanisms in detail, research has broadly focused in the following areas: (1) immune system dysfunction, associated with a proinflammatory state and impaired white blood cell function; (2) metabolic derangements leading to oxidative stress, release of free fatty acids, reduction in endogenous insulin secretion, and fluid and electrolyte imbalance; and (3) a wide variety of vascular system responses (eg, endothelial dysfunction with impairment of tissue perfusion, a prothrombotic state, increased platelet aggregation, and left ventricular dysfunction). ${ }^{8,16-18}$

Conversely administration of insulin suppresses or reverses many of these abnormalities including generation of reactive oxygen species (ROS) and activation of inflammatory mechanisms, ${ }^{19}$ and leads to a fall in C-reactive protein, which accompanied the clinical benefit of intensive insulin therapy (IIT) in the Leuven, Belgium, ICU population, ${ }^{20}$ and prevents mitochondrial abnormalities in hepatocytes. ${ }^{21}$ In the same surgical ICU cohort, Langouche et al. ${ }^{22}$ report suppression of intracellular adhesion molecule-1 (ICAM1) and E-selectin, markers of inflammation, and reduction in plasma nitric oxide (NO) and innate nitric oxide (iNOS) expression with insulin administration in patients treated with intravenous (IV) IIT. $^{22}$ These data further support the role of insulin infusion in suppressing inflammation and endothelial dysfunction. The authors suggest that maintaining normoglycemia with IIT during critical illness protects the endothelium, thereby contributing to prevention of organ failure and death. ${ }^{22}$ Based on accumulating data in the literature such as that cited above, it has been suggested that a new paradigm in which glucose and insulin are related not only through their metabolic action but also through inflammatory mechanisms offers important potential therapeutic opportunities. ${ }^{19}$

\section{Evidence: Epidemiology/Observational Studies/Non-RCT Interventional Studies}

A strong association between hospital hyperglycemia and negative outcomes has been reported in numerous observational studies in diverse adult medical and surgical settings. In over 1800 hospital admissions, those with new hyperglycemia had an in-hospital mortality rate of $16 \%$ compared with $3 \%$ mortality in patients with known diabetes 
and $1.7 \%$ in normoglycemic patients $(P<0.01)$. These data suggest that hyperglycemia due to previously unrecognized diabetes may be an independent marker of in-hospital mortality. ${ }^{13}$

Hyperglycemia has been linked to adverse outcomes in myocardial infarction, stroke, ${ }^{23-28}$ postoperative nosocomial infection risk, pneumonia, renal transplant, cancer chemotherapy, percutaneous coronary interventions, and cardiac surgery. ${ }^{29-38}$ These observational studies have the usual limitations inherent in their design. Demonstrating a strong association of hyperglycemia with adverse outcomes is not a guarantee that the hyperglycemia is the cause for the poor outcome, as hyperglycemia can reflect a patient under more stress who is at a higher risk for adverse outcome. By the same token, the strong association of hyperglycemia with the risk of poor outcomes seen in these studies does not guarantee that euglycemia would mitigate this risk.

Nonetheless, there are several factors that make the body of evidence for glycemic control more compelling. First, the association has a rational physiologic basis as described above. Second, the associations are consistent across a variety of patient populations and disease entities, and demonstrate a dose-response relationship. Third, in studies that control for comorbidities and severity of illness, hyperglycemia persists as an independent risk factor for adverse outcomes, whether the patient has a preexisting diagnosis of diabetes or not. Last, non-RCT interventional studies and RCTs largely reinforce these studies.

The Portland Diabetic Project has reported prospective, nonrandomized data over 17 years on the use of an IV insulin therapy protocol in cardiac surgery patients. ${ }^{38}$ This program has implemented stepped lowering of target BG, with the most recent data report implementing a goal BG $<150 \mathrm{mg} / \mathrm{dL}^{35}$ The current protocol uses a BG target of $70-110 \mathrm{mg} / \mathrm{dL}$, but results have not yet been published. ${ }^{39}$ Mortality and deep sternal wound infection rates for patients with diabetes who remain on the IV insulin protocol for 3 days have been lowered to levels equivalent to those for nondiabetic patients. This group has also reported reductions in length of stay and costeffectiveness of targeted glycemic control in the cardiac surgery population. ${ }^{35}$ Their data have to a large extent driven a nationwide movement to implement targeted BG control in cardiac surgery patients.
Another large ICU study (mixed medical-surgical, $\mathrm{n}=800$ patients) also supports a benefit through targeted BG control (130.7 versus 152.3 $\mathrm{mg} / \mathrm{dL}, P<0.001)$ when compared with historical controls. This study demonstrated reduction in inhospital mortality (relative risk reduction $29.3 \%, P$ $=0.002)$, duration of ICU stay $(10.8 \%, P=0.04)$, acute renal failure $(75 \%, P=0.03)$, and blood transfusions $(18.7 \%, P=0.002),{ }^{40}$ representing a similar magnitude of effect as was demonstrated by the Belgian group.

\section{Evidence: RCTs}

Evidence is accumulating that demonstrates an advantage in terms of morbidity and mortality when targeted glycemic control using intravenous insulin infusion is implemented in the hospital. The most robust data have been reported from ICU and cardiac surgery settings. The largest randomized, controlled study to date enrolled 1548 patients in a surgical ICU in Leuven, Belgium who were randomized to either intensive (IT) or conventional (CT) insulin therapy. Mean glucose attained was $103 \pm 19$ and $153 \pm 33 \mathrm{mg} / \mathrm{dL}$ in each arm, respectively. The intensive insulin group demonstrated a reduction in both ICU (4.6\% versus $8.0 \%)$ and in-hospital mortality $(7.2 \%$ versus $10.9 \%)$, as well as bloodstream infections, acute renal failure, transfusions, and polyneuropathy, the latter being reflected by duration of mechanical ventilation $(P<0.01$ for all). Although a similar study in an MICU did not achieve statistical significance in the overall intention-to-treat analysis, it did demonstrate reductions in mortality (from $52.5 \%$ to $43.0 \%$ ) in patients with at least 3 days of ICU treatment. It should also be noted that in this MICU population hypoglycemia rates were higher and level of glycemic control attained not as rigorous as in the same group's SICU cohort, factors which may have had an impact on observed outcomes. A meta-analysis of these two Leuven, Belgium, studies demonstrated a reduction in mortality $(23.6 \%$ versus $20.4 \%$, absolute risk reduction [ARR] $3.2 \%, P=0.004)$ ) in all patients treated with IIT, with a larger reduction in mortality (37.9\% versus $30.1 \%$, ARR $7.8 \%, \quad P=0.002$ ) observed in patients with at least 3 days of IIT, as well as substantial reductions in morbidity. ${ }^{9,10,41,42}$

Several other studies must be mentioned in this context. A small $(\mathrm{n}=61)$, randomized study in another SICU did not show a mortality benefit, perhaps because the number of subjects was not 
adequate to reach statistical significance, but did result in a significant reduction in nosocomial infections in patients receiving IIT (BG $=125$ versus $179 \mathrm{mg} / \mathrm{dL}, P<0.001) .{ }^{43}$ Two international multicenter studies recently stopped enrollment due to excess rates of hypoglycemia. The Volume Substitution and Insulin Therapy in Severe Sepsis (VISEP) study, in a mixed medical and surgical sepsis population, showed no significant reduction in mortality in the intensively-treated group. Serious adverse events were reported according to standard definitions. Enrollment was stopped before the full number of subjects had been randomized. Among the 537 evaluable cases, hypoglycemia (BG $<40 \mathrm{mg} / \mathrm{dL}$ ) was reported as $17.0 \%$ in the IT group and $4.1 \%(P<0.001)$ in the control group, ${ }^{44}$ and the rate of serious adverse events was higher in the IT group $(10.9 \%$ versus $5.2 \%, P$ $=0.01)$. It is notable that the rate of hypoglycemia was comparable to the $18.7 \%$ rate seen in the IT group in the Leuven, Belgium, medical ICU study. ${ }^{9}$ The Glucontrol study enrolled 855 medical and surgical ICU patients and was similarly terminated because of hypoglycemia ( $\mathrm{BG}<40 \mathrm{mg} / \mathrm{dL}$ ) at a rate of $8.6 \%$ compared to $2.4 \%$ in the control group $(P<0.001)$. Insulin infusion protocols and outcome data have not yet been published. ${ }^{42,45}$

These studies with very high hypoglycemia rates each used an algorithm based on the Leuven, Belgium, protocol. The rates of severe hypoglycemia are $3-4 \times$ that reported by a variety of others achieving similar or identical glycemic targets. Hypoglycemia should not be construed as a reason to not use a standardized insulin infusion protocol. In comparing protocols that have been published, it is apparent that rates of hypoglycemia differ substantially and that performance results of some algorithms are not necessarily replicable across sites. ${ }^{46}$ Dose-defining designs can be substantively more sophisticated than those used in the trials mentioned, in some cases incorporating principles of control engineering. The variability of hypoglycemia rates under differing insulin infusion protocols is a compelling reason to devote institutional effort to monitoring the efficacy and safety of the infusion protocols that are used.

High-level evidence from randomized, controlled trials demonstrating outcomes benefit through targeted BG control outside the ICU is lacking at this point in time, but it must be noted that feasibility is suggested by a recent randomized control trial (RABBIT2) that demonstrated the superiority of basal bolus insulin regimens to sliding scale insulin in securing glycemic control, without any increase in hypoglycemia. ${ }^{47}$

\section{Summing Up the Evidence}

It is clear that hyperglycemia is associated with negative clinical outcomes throughout the hospital, and level A evidence is available to support tight glucose control in the SICU setting. However, in view of the imperfect and incomplete nature of the evidence, controversy persists around how stringent glycemic targets should be in the ICU, on whether glycemic targets should differ between SICU and MICU patients, and especially what the targets should be in the non-ICU setting. There should be hesitancy to extrapolate glycemic targets to be applied beyond the populations that have been studied with RCTs or to assume benefit for medical conditions that have not been examined for the impact of interventions to control hyperglycemia. Institutions might justifiably choose more liberal targets than those promoted in national recommendations/guidelines ${ }^{2,48-50}$ until safe attainment of more moderate goals is demonstrated. However, even critics agree that uncontrolled hyperglycemia exceeding $180-200 \mathrm{mg} / \mathrm{dL}$ in any acute care setting is undesirable. Moreover, strong observational data showing the hazards of hyperglycemia in noncritical care units (even after adjustment for severity of illness) combined with the high rate of adverse drug events associated with insulin use, argue strongly for a standardized approach to treating diabetes and hyperglycemia in the hospital. Even though no RCTs exist demonstrating outcomes benefits of achieving glycemic target on wards, the alternatives to control of hyperglycemia using scheduled insulin therapy are unacceptable. Oral agent therapy is potentially dangerous and within the necessary timeframe is likely to be ineffective; sliding scale management is inferior to basal-bolus insulin therapy, as shown in an $\mathrm{RCT}^{47}$ and is unsafe; and on the wards improved glycemic control can be achieved simultaneously with a reduction in hypoglycemia. ${ }^{51}$

\section{INPATIENT GLYCEMIC CONTROL IS INCREASINGLY INCORPORATED INTO PUBLIC REPORTING, GUIDELINES, REGULATORY AGENCY, AND NATIONAL QUALITY INITIATIVE PRIORITIES}

National quality initiatives, public reporting, payfor-performance, and guideline-based care con- 
tinue to play an increasingly important role in the U.S. healthcare system. Over the years these initiatives have focused on various disease states (venous thromboembolism, congestive heart failure, community-acquired pneumonia, etc.) in an attempt to standardize care and improve patient safety and quality. Inpatient hyperglycemic control is also increasingly being incorporated into public reporting, regulatory compliance, and national quality initiatives.

Professional organizations such as the $\mathrm{ADA}^{2}$ and $\mathrm{AACE}^{50}$ have published guidelines supporting improved glycemic control, the safe use of insulin, and other measures to improve care for hyperglycemic inpatients. The AACE has a Web site dedicated to hospital hyperglycemia. ${ }^{52}$ The Society of Hospital Medicine ${ }^{48}$ has created a resource room on its Web site and a workbook for improvement ${ }^{49}$ on optimizing the care of inpatients with hyperglycemia and diabetes. The guidelines and Web sites help raise awareness and educate physicians and healthcare workers in inpatient glucose management. The American Heart Association has incorporated specific recommendation regarding inpatient diabetic management in its "Get With the Guidelines." 53

The Joint Commission ${ }^{54}$ has developed an advanced disease-specific certification on inpatient diabetes. Disease management programs are important components of complex healthcare systems that serve to coordinate chronic care, promote early detection and prevention, and reduce overall healthcare costs. Certification is increasingly important to providers, payers, and healthcare institutions because it demonstrates a commitment to quality and patient safety. The Joint Commission disease-specific care certification is a patient-centered model focusing on the delivery of clinical care and relationship between the practitioner and the patient. The evaluation and resulting certification by the Joint Commission is based on 3 core components: (1) an assessment of compliance with consensus-based national standards; (2) the effective use of established clinical practice guidelines to manage and optimize care; and (3) an organized approach to performance measurement and improved activities. ${ }^{55}$ For inpatient diabetes, the Joint Commission program has 7 major elements following the ADA recommendations, including general recommendations regarding diabetic documentation, BG targets, preventing hypoglycemia, diabetes care providers, diabetes self-management education, medical nutrition therapy, and BG monitoring. ${ }^{54}$ This mirrors the Call to Action Consensus Conference essential elements for successful glycemic control programs. ${ }^{1}$

Other organizations such as the Surgical Care Improvement Partnership (SCIP) and National Surgical Quality Improvement Program (NSQIP) have included perioperative glycemic control measures, as it impacts surgical wound infections. The University HealthSystem Consortium (UHC) has benchmarking data and endorses perioperative glycemic control measures, whereas the Institute for Healthcare Improvement (IHI) has focused on safe use of insulin practices in its 5 Million Lives campaign.

\section{HOSPITALIZATION IS A MOMENT OF OPPORTUNITY TO ASSESS AND INTERVENE}

The benefits of outpatient glycemic control and quality preventive care are well established, and the reduction of adverse consequences of uncontrolled diabetes are a high priority in ambulatory medicine. ${ }^{56-58}$ Hospitalization provides an opportunity to identify previously undiagnosed diabetes or prediabetes and, for patients with known diabetes, to assess and impact upon the long term course of diabetes.

As a first step, unless a recent hemoglobin A1C (HbAlc) is known, among hospitalized hyperglycemic patients an HbAlC should be obtained upon admission. Greci et al. ${ }^{59}$ showed that an HbAlc level $>6.0 \%$ was $100 \%$ specific (14/14) and $57 \%$ sensitive (12/21) for the diagnosis of diabetes. Among patients having known diabetes, an HbAlC elevation on admission may justify intensification of preadmission management at the time of discharge. If discharge and postdischarge adjustments of preadmission regimens are planned in response to admission A1C elevations, then the modified long-term treatment strategy can improve the AlC in the ambulatory setting. ${ }^{60}$ Moreover, the event of hospitalization is the ideal "teachable moment" for patients and their caregivers to improve self-care activities. Yet floor nurses may be overwhelmed by the tasks of patient education. For ideal patient education, both a nutritionist and a diabetes nurse educator are needed to assess compliance with medication, diet, and other aspects of care. ${ }^{61-63}$ There also is need for outpatient follow-up education. Finally, at the time of discharge, there is a 
duty and an opportunity for the diabetes provider to communicate with outpatient care providers about the patient's regimen and glycemic control, and also, based on information gathered during the admission, to convey any evidence that might support the need for a change of long-term strategy. ${ }^{64}$ Unfortunately, the opportunity that hospitalization presents to assess, educate, and intervene frequently is underused. ${ }^{1,8,51,65}$

\section{LARGE GAPS EXIST BETWEEN CURRENT AND OPTIMAL CARE}

Despite the evidence that inpatient glycemic control is important for patient outcomes, and despite guidelines recommending tighter inpatient glycemic control, clinical practice has been slow to change. In many institutions, inpatient glycemic management has not improved over the past decade, and large gaps remain between current practice and optimal practice.

Studies of individual institutions provide several insights into gaps in care. For example, Schnipper et al. ${ }^{66}$ examined practices on the general medicine service of an academic medical center in Boston in 2004. Among 107 prospectively identified patients with a known diagnosis of diabetes or at least 1 glucose reading $>200 \mathrm{mg} / \mathrm{dL}$ (excluding patients with diabetic ketoacidosis, hyperglycemic hyperosmolar state, or pregnancy), they found scheduled long-acting insulin prescribed in $43 \%$ of patients, scheduled short-acting/rapid-acting insulin in only $4 \%$ of patients, and 80 of 89 patients $(90 \%)$ on the same sliding scale insulin regimen despite widely varying insulin requirements. Thirty-one percent of glucose readings were $>180 \mathrm{mg} / \mathrm{dL}$ compared with $1.2 \%$ of readings $<60 \mathrm{mg} / \mathrm{dL}$ (but $11 \%$ of patients had at least 1 episode of hypoglycemia). Of the 75 patients with at least 1 episode of hyperglycemia or hypoglycemia, only $35 \%$ had any change to their insulin regimen during the first 5 days of the hospitalization.

Other studies have confirmed this concept of clinical inertia (ie, recognition of the problem but failure to act). ${ }^{67} \mathrm{~A}$ study by Cook et al. ${ }^{68}$ of all hospitalized non-ICU patients with diabetes or hyperglycemia and length of stay of 3 days between 2001 and 2004 showed that $20 \%$ of patients had persistent hyperglycemia during the hospitalization (defined as a mean glucose $>200 \mathrm{mg} / \mathrm{dL}$ ). Forty-six percent of patients whose average glucose was in
TABLE 1

Results of the University HealthSystem Consortium

Benchmarking Project

\begin{tabular}{ll}
\hline Key Performance Measure & $\begin{array}{l}\text { Results for Median-Performing } \\
\text { Hospital (\%) }\end{array}$ \\
\hline $\begin{array}{l}\text { Documentation of diabetes } \\
\text { Hob Alc assessment within } 30 \text { days }\end{array}$ & 100 \\
Glucose measurement within 8 hours & 36.1 \\
$\quad$ of admission & 78.6 \\
Glucose monitoring $\geq 4$ times a day & 85.4 \\
Median glucose reading $>200 \mathrm{mg} / \mathrm{dL}$ & 10.3 \\
Effective insulin therapy* & 44.7 \\
ICU day 2 morning glucose $\leq 110 \mathrm{mg} / \mathrm{dL}$ & 17.7 \\
Non-ICU day 2 all glucose readings & \\
$\quad \leq 180 \mathrm{mg} / \mathrm{dL}$ & 26.3 \\
Patient-days with at least 1 glucose & \\
reading $<50 \mathrm{mg} / \mathrm{dL}$ & 2.4 \\
\hline
\end{tabular}

Abbreviation: ICU, intensive care unit.

${ }^{*}$ Combination of short-acting/rapid-acting and long-acting subcutaneous insulins, continuous insulin infusion, or subcutaneous insulin pump.

the top tertile did not have their insulin regimen intensified to a combination of short-acting/rapidacting and long-acting insulin, and 35\% of these patients either had no change in their total daily insulin dose or actually had a decrease in their dose when comparing the last 24 hours with the first 24 hours of hospitalization, a concept they term "negative therapeutic momentum."

Perhaps the most well-balanced view of the current state of medical practice comes from the UHC benchmarking project. ${ }^{69} \mathrm{UHC}$ is an alliance of 90 academic health centers. For the diabetes project, each institution reviewed the records of 50 randomly selected patients over 18 years of age with at least a 72-hour length of stay, 1 of 7 prespecified Diagnosis Related Group (DRG) codes, and at least 2 consecutive glucose readings $>180$ $\mathrm{mg} / \mathrm{dL}$ or the receipt of insulin any time during the hospitalization. Patients with a history of pancreatic transplant, pregnant at the time of admission, receiving hospice or comfort care, or receiving insulin for a reason other than glucose management were excluded. The study showed widespread gaps in processes and outcomes (Table 1). Moreover, performance varied widely across hospitals. For example, the morning glucose in the ICU on the second measurement day was $110 \mathrm{mg} / \mathrm{dL}$ in $18 \%$ of patients for the medianperforming hospital, with a range of $0 \%$ to $67 \%$ across all 37 measured hospitals. In the non-ICU setting on the second measurement day, $26 \%$ of 
patients had all BG measurements $=180 \mathrm{mg} / \mathrm{dL}$ in the median-performing hospital, with a range of $7 \%$ to $48 \%$. Of note, hypoglycemia was relatively uncommon: in the median hospital, $2.4 \%$ of patient-days had 1 or more BG readings $<50 \mathrm{mg} /$ dL (range: 0\%-8.6\%). Finally, in the median-performing hospital, effective insulin therapy (defined as short-acting/rapid-acting and long-acting subcutaneous insulin, continuous insulin infusion, or subcutaneous insulin pump therapy) was prescribed in $45 \%$ of patients, with a range of $12 \%$ to $77 \%$ across measured hospitals.

\section{FREQUENT PROBLEMS WITH COMMUNICATION AND COORDINATION}

Those who work closely with frontline practitioners striving to improve inpatient glycemic management have noticed other deficiencies in care. $^{1,70}$ These include: a lack of coordination between feeding, BG measurement, and insulin administration, leading to mistimed and incorrectly dosed insulin; frequent use of sliding-scale only regimens despite evidence that they are useless at best and harmful at worst; ${ }^{6,47,60,71}$ discharge summaries that often do not mention follow-up plans for hyperglycemic management; incomplete patient educational programs; breakdowns in care at transition points; nursing and medical staffs that are unevenly educated about the proper use of insulin; and patients who are often angry or confused about their diabetes care in the hospital. Collectively, these gaps in care serve as prime targets for any glycemic control program.

\section{HYPOGLYCEMIA IS A PROMINENT INPATIENT SAFETY CONCERN}

Hypoglycemia is common in the inpatient setting and is a legitimate safety concern. In a recently reported series of 2174 hospitalized patients receiving antihyperglycemic agents, it was found that $9.5 \%$ of patients experienced a total 484 hypoglycemic episodes (defined as $\leq 60 \mathrm{mg} / \mathrm{dL}$ ) ${ }^{72}$ Hypoglycemia often occurred in the setting of insulin therapy and frequently resulted from a failure to recognize trends in BG readings or other clues that a patient was at risk for developing hypoglycemia. ${ }^{73} \mathrm{~A}$ common thread is the risk created by interruption of carbohydrate intake, noted by Fischer et al. ${ }^{73}$ and once again in the recent ICU study by Vriesendorp et al. $^{74}$ Sources of error include: lack of coordination between feeding and medication administra- tion, leading to mistiming of insulin action; lack of sufficient frequency in BG monitoring; lack of clarity or uniformity in the writing of orders; failure to recognize changes in insulin requirements due to advanced age, renal failure, liver disease, or change in clinical status; steroid use with subsequent tapering or interruption; changes in feeding; failure to reconcile medications; inappropriate use of oral antihyperglycemic agents, and communication or handoff failures.

It has been difficult to sort out whether hypoglycemia is a marker of severity of illness or whether it is an independent factor leading to poor outcomes. Observational studies lend credibility to the concept that patients having congestive heart failure or myocardial infarction may be at risk for excessive mortality if their average BG resides in the low end of the normal range. ${ }^{75-78}$ Sympathetic activation occurs as the threshold for hypoglycemia is approached, such as occurs at BG $=70$ or $72 \mathrm{mg} / \mathrm{dL} .{ }^{79}$ Patients living with BG levels observed to be in the low end of the normal range might experience more severe but unobserved and undocumented episodes of neuroglycopenia. Arrhythmia and fatality have been directly attributed to strict glycemic control. ${ }^{80,81}$ We are confronted with the need to interpret well conducted observational studies, evaluating subgroups at risk, and using multivariate analysis to assess the impact of hypoglycemia upon outcomes. ${ }^{82}$ In such studies, we will need to examine high-risk subgroups, including cardiac patients, in particular, for the possibility that there is a J-shaped curve for mortality as a function of average BG.

Unfortunately, clinical inertia exists in response to hypoglycemia just as it does with hyperglycemia. One recent study examined 52 patients who received intravenous $50 \%$ dextrose solution for an episode of hypoglycemia. ${ }^{83}$ Changes to insulin regimens were subsequently made in only 21 patients $(40 \%)$, and diabetes specialists agreed with the changes for 11 of these patients. The other 31 patients $(60 \%)$ received no changes in treatment, and diabetes specialists agreed with that decision for only 10 of these patients.

Although some increase in hypoglycemia might be expected with initiation of tight glycemic control efforts, the solution is not to undertreat hyperglycemia. Hyperglycemia creates an unsafe setting for the treatment of illness and disease. Sliding-scale-only regimens are ineffective in securing glycemic control and can result in 
increases in hypoglycemia as well as hyperglycemic excursions. ${ }^{6,66}$ Inappropriate withholding of insulin doses can lead to severe glycemic excursions and even iatrogenic diabetic ketoacidosis (DKA). Systems approaches to avoid the errors outlined above can minimize or even reverse the increased risk of hypoglycemia expected with tighter glycemic targets. ${ }^{51}$

\section{A SYSTEMS APPROACH IS NEEDED FOR THESE MULTIPLE COMPLEX PROBLEMS}

Care is of the hyperglycemic inpatient is inherently complex. Previously established treatments are often inappropriate under conditions of altered insulin resistance, changing patterns of nutrition and carbohydrate exposure, comorbidities, concomitant medications, and rapidly changing medical and surgical status. Patients frequently undergo changes in the route and amount of nutritional exposure, including discrete meals, continuous intravenous dextrose, nil per orem (nothing by mouth status; NPO) status, grazing on nutritional supplements or liquid diets with or without meals, bolus enteral feedings, overnight enteral feedings with daytime grazing, total parenteral nutrition, continuous peritoneal dialysis, and overnight cycling of peritoneal dialysis. Relying on individual expertise and vigilance to negotiate this complex terrain without safeguards, protocols, standardization of orders, and other systems change is impractical and unwise.

Transitions across care providers and locations lead to multiple opportunities for breakdown in the quality, consistency, and safety of care ${ }^{64,65}$ At the time of ward transfer or change of patient status, previous medication and monitoring orders sometimes are purged. At the time of discharge, there may be risk of continuation of anti-hyperglycemic therapy, initiated to cover medical stress, in doses that will subsequently be unsafe.

In the face of this complexity, educational programs alone will not suffice to improve care. Institutional commitment and systems changes are essential.

\section{MARKED IMPROVEMENT IS POSSIBLE AND TOOLS EXIST: A ROADMAP IS IN PLACE}

Fortunately, a roadmap is in place to help us achieve better glycemic control, improve insulin management, and address the long list of current deficiencies in care. This is imperative to develop consistent processes in order to achieve maximum patient quality outcomes that effective glycemic management offers. This roadmap entails 4 components: (1) national awareness, (2) national guidelines, (3) consensus statements, and (4) effective tools. As mentioned above, the first two components of this roadmap are now in place.

As these national guidelines become more widely accepted, the next step will be the incorporation of this into programs like Pay-for Performance and the Physician Quality Reporting Initiative (PQRI), which will impact reimbursement to both hospitals and providers.

Regarding the third component, a recent multidisciplinary consensus conference ${ }^{1}$ outlined the essential elements needed for successful implementation of an inpatient glycemic control program which include:

- An appropriate level of administrative support.

- Formation of a multidisciplinary steering committee to drive the development of initiatives and empowered to enact change.

- Assessment of current processes, quality of care, and barriers to practice change.

- Development and implementation of interventions including standardized order sets, protocols, policies and algorithms with associated educational programs.

- Metrics for evaluation of glycemic control, hypoglycemia, insulin use patterns, and other aspects of care.

Finally, extensive resources and effective tools are now available to help institutions achieve better inpatient glucose control. The Society of Hospital Medicine (SHM), in conjunction with the ADA, AACE, the American College of Physicians (ACP), the Case Management Society of America (CMSA), the American Society of Consultant Pharmacists, nursing, and diabetic educators have all partnered to produce a comprehensive guide to effective implementation of glycemic control and preventing hypoglycemia. ${ }^{49}$ This comprehensive workbook is a proven performance improvement framework and is available on the SHM Web site. ${ }^{48}$ Details and examples of all essential elements are covered in this workbook along with opportunities for marked improvement bolstered by integration of high reliability design features and attention to effective implementation techniques. The remainder of this supplement crystallizes a substantial portion of this material. The 
AACE has also recently offered a valuable webbased resource to encourage institutional glycemic control efforts. ${ }^{49}$

\section{GLYCEMIC CONTROL INITIATIVES CAN BE COST-EFFECTIVE}

Achieving optimal glycemic control safely requires monitoring, education, and other measures, which can be expensive, labor intensive, and require coordination of the services of many hospital divisions. This incremental expense has been shown to be cost-effective in a variety of settings. ${ }^{1,84,85}$ The costs of glycemic control initiatives have demonstrated a good return on investment via:

- Improved LOS, readmission rates, morbidity, and mortality.

- Improved documentation of patient acuity and related payment for acuity.

- Income generated via incremental physician and allied health professional billing.

\section{CONCLUSION AND SUMMARY}

Evidence exists that appropriate management of hyperglycemia improves outcomes, whereas the current state of affairs is that most medical centers currently manage this suboptimally. This is concerning given the magnitude of diabetes and hyperglycemia in our inpatient setting in the United States. To bring awareness to this issue, multiple initiatives (guidelines, certification programs, workbooks, etc.) are available by various organizations including the ADA, AACE, SCIP, NSQIP, IHI, UHC, the Joint Commission, and SHM. However, this is not enough. Change occurs at the local level, and institutional prioritization and support is needed to empower a multidisciplinary steering committee, with appropriate administrative support, to standardize and improve systems in the face of substantial cultural issues and complex barriers. Improved data collection and reporting, incremental monitoring, creation of metrics, and improved documentation are an absolutely necessary necessity to achieve breakthrough levels of improvement.

Now the time is right to make an assertive effort to improve inpatient glycemic control and related issues, and push for appropriate support at your institution to help achieve this in the interest of patient safety and optimal outcomes.

Address for correspondence and reprint requests: Susan S. Braithwaite, MD, FACP, FACE, Clinical Professor of Medicine, University of North Carolina at Chapel Hill, Chapel Hill, NC 27599; E-mail: sbraithw@med.unc.edu
Received 10 January 2008; revision received 13 July 2008; accepted 17 July 2008.

\section{REFERENCES}

1. American College of Endocrinology and American Diabetes Association Consensus Statement on Inpatient Diabetes and Glycemic Control: A call to action. Diabetes Care. 2006; 29:1955-1962.

2. Standards of medical care in diabetes--2008. Diabetes Care. 2008;31(Suppl 1):S12-S54.

3. Cowie CC, Rust KF, Byrd-Holt DD, et al. Prevalence of diabetes and impaired fasting glucose in adults in the U.S. population: National Health And Nutrition Examination Survey 1999-2002. Diabetes Care. 2006;29:1263-1268.

4. Centers for Disease Control and Prevention. National Diabetes Fact Sheet: General Information and National Estimates on Diabetes in the United States, 2005. Atlanta, GA: U.S. Department of Health and Human Services, Centers for Disease Control and Prevention, 2005. Available at: http://www.cdc.gov/diabetes/pubs/factsheet05.htm. Accessed September 2007.

5. Hogan P, Dall T, Nikolov P. Economic costs of diabetes in the US in 2002. Diabetes Care. 2003;26:917-932.

6. Queale WS, Seidler AJ, Brancati FL. Glycemic control and sliding scale insulin use in medical inpatients with diabetes mellitus. Arch Intern Med. 1997;157:545-552.

7. Metchick LN, Petit WA Jr, Inzucchi SE. Inpatient management of diabetes mellitus. Am J Med. 2002;113:317-323.

8. Clement S, Braithwaite SS, Magee MF, et al. Management of diabetes and hyperglycemia in hospitals. Diabetes Care. 2004;27:553-591.

9. Van den Berghe G, Wilmer A, Hermans G, et al. Intensive insulin therapy in the medical ICU. $N$ Engl J Med. 2006; 354:449-461.

10. Van den Berghe G, Wouters P, Weekers F, et al. Intensive insulin therapy in critically ill patients. $N$ Engl J Med. 2001; 345:1359-1367.

11. Krinsley JS. Association between hyperglycemia and increased hospital mortality in a heterogeneous population of critically ill patients. Mayo Clin Proc. 2003;78:1471-1478.

12. Levetan CS, Passaro M, Jablonski K, Kass M, Ratner RE. Unrecognized diabetes among hospitalized patients. Diabetes Care. 1998;21:246-249.

13. Umpierrez GE, Isaacs SD, Bazargan N, You X, Thaler LM, Kitabchi AE. Hyperglycemia: an independent marker of inhospital mortality in patients with undiagnosed diabetes. J Clin Endocrinol Metab. 2002;87:978-982.

14. United States Department of Health and Human Services Agency for Healthcare Research and Quality. 2007. Available at: http://hcupnet.ahrq.gov. Accessed December 2007.

15. Norhammar A, Tenerz A, Nilsson G, et al. Glucose metabolism in patients with acute myocardial infarction and no previous diagnosis of diabetes mellitus: a prospective study. Lancet. 2002;359:2140-2144.

16. Zarich SW. Mechanism by which hyperglycemia plays a role in the setting of acute cardiovascular illness. Rev Cardiovasc Med. 2006;7(Suppl 2):S35-S43.

17. Bauters C, Ennezat PV, Tricot O, Lauwerier B, Lallemant R, Saadouni H, et al. Stress hyperglycaemia is an independent predictor of left ventricular remodelling after first anterior myocardial infarction in non-diabetic patients. Eur Heart J. 2007;28:546-552. 
18. Zarich SW, Nesto RW. Implications and treatment of acute hyperglycemia in the setting of acute myocardial infarction. Circulation. 2007;115:e436-e439.

19. Dandona P, Mohanty P, Chaudhuri A, Garg R, Aljada A. Insulin infusion in acute illness. J Clin Invest. 2005;115: 2069-2072.

20. Hansen T, Thiel S, Wouters P, Christiansen J, Van den Berghe B. Intensive insulin therapy exerts antiinflammatory effects in critically ill patients and counteracts the adverse effect of low mannose-gind lectin levels. J Clin Endocrinol Metab. 2003;88:1082-1088.

21. Vanhorebeek I, De Vos R, Mesotten D, Wouters PJ, De Wolf-Peeters C, Van den Berghe G. Protection of hepatocyte mitochondrial ultrastructure and function by strict blood glucose control with insulin in critically ill patients. Lancet. 2005;365:53-59.

22. Langouche L, Vanhorebeek I, Vlasselaers D, et al. Intensive insulin therapy protects the endothelium of critically ill patients. J Clin Invest. 2005;115:2277-2286.

23. Ainla T, Baburin A, Teesalu R, Rahu M. The association between hyperglycaemia on admission and 180-day mortality in acute myocardial infarction patients with and without diabetes. Diabet Med. 2005;22:1321-1325.

24. Kosiborod M, Rathore SS, Inzucchi SE, et al. Admission glucose and mortality in elderly patients hospitalized with acute myocardial infarction: implications for patients with and without recognized diabetes. Circulation. 2005;111: 3078-3086.

25. Malmberg K, Norhammar A, Wedel H, Ryden L. Glycometabolic state at admission: important risk marker of mortality in conventionally treated patients with diabetes mellitus and acute myocardial infarction: long-term results from the Diabetes and Insulin-Glucose Infusion in Acute Myocardial Infarction (DIGAMI) study. Circulation. 1999; 99:2626-2632.

26. Capes S, Hunt D, Malmberg K, Gerstein H. Stress hyperglycaemia and increased risk of death after myocardial infarction in patients with and without diabetes: a systematic overview. Lancet. 2000;355:773-778.

27. Bruno A, Williams LS, Kent TA. How important is hyperglycemia during acute brain infarction? Neurologist. 2004; 10:195-200.

28. Capes S, Hunt D, Malmberg K, Pathak P, Gerstein H. Stress hyperglycemia and prognosis of stroke in nondiabetic and diabetic patients: a systematic overview. Stroke. 2001;32: 2426-2432.

29. Golden SH, Peart-Vigilance C, Kao WHL, Brancati F. Perioperative glycemic control and the risk of infectious complications in a cohort of adults with diabetes. Diabetes Care. 1999;22:1408-1414.

30. Pomposelli JJ, Baxter JK, Babineau TJ, et al. Early postoperative glucose control predicts nosocomial infection rate in diabetic patients. JPEN J Parenter Enteral Nutr. 1998;22:77-81.

31. McAlister FA, Majumdar SR, Blitz S, Rowe BH, Romney J, Marrie TJ. The relation between hyperglycemia and outcomes in 2,471 patients admitted to the hospital with communityacquired pneumonia. Diabetes Care. 2005;28:810-815.

32. Thomas M, Mathew T, Russ G, Rao M, Moran J. Early perioperative glycaemic control and allograft rejection in patients with diabetes mellitus: a pilot study. Transplantation. 2001;72:1321-1324.
33. Weiser MA, Cabanillas ME, Konopleva M, et al. Relation between the duration of remission and hyperglycemia during induction chemotherapy for acute lymphocytic leukemia with a hyperfractionated cyclophosphamide, vincristine, doxorubicin, and dexamethasone/methotrexate-cytarabine regimen. Cancer. 2004;100:1179-1185.

34. Muhlestein JB, Anderson JL, Horne BD, et al. Effect of fasting glucose levels on mortality rate in patients with and without diabetes mellitus and coronary artery disease undergoing percutaneous coronary intervention. Am Heart J. 2003;146:351-358.

35. Furnary AP, Wu Y, Bookin SO. Effect of hyperglycemia and continuous intravenous insulin infusions on outcomes of cardiac surgical procedures: the Portland diabetic project. Endocr Pract. 2004;10(Suppl 2):21-33.

36. Gandhi GY, Nuttall GA, Abel MD, et al. Intraoperative hyperglycemia and perioperative outcomes in cardiac surgery patients. Mayo Clin Proc. 2005;80:862-866.

37. Latham R, Lancaster AD, Covington JF, Pirolo JS, Thomas CS. The association of diabetes and glucose control with surgical-site infections among cardiothoracic surgery patients. Infect Control Hosp Epidemiol. 2001;22:607-612.

38. Zerr KJ, Furnary AP, Grunkemeier GL. Glucose control lowers the risk of wound infection in diabetics after open heart operations. Ann Thorac Surg. 1997;63:356-361.

39. The Portland Protocol. Available at: http://www.providence. org/oregon/grograms_and_services/heart/portlandprotocol/. Accessed September 2007.

40. Krinsley JS. Effect of an intensive glucose management protocol on the mortality of critically ill adult patients. Mayo Clin Proc. 2004;79:992-1000.

41. Van den Berghe G, Wilmer A, Milants I, et al. Intensive insulin therapy in mixed medical/surgical intensive care units: benefit versus harm. Diabetes. 2006;55:3151-3159.

42. Vanhorebeek I, Langouche L, Van den Berghe G. Tight blood glucose control with insulin in the ICU: facts and controversies. Chest. 2007;132:268-278.

43. Grey NJ, Perdrizet GA. Reduction of nosocomial infections in the surgical intensive-care unit by strict glycemic control. Endocr Pract. 2004;10(Suppl 2):46-52.

44. Brunkhorst FM, Engel C, Bloos F, Meier-Hellmann A, Ragaller $\mathrm{M}$, Weiler $\mathrm{N}$, et al. Intensive insulin therapy and pentastarch resuscitation in severe sepsis. $N$ Engl J Med. 2008; 358:125-139.

45. Devos P, Preiser JC. Current controversies around tight glucose control in critically ill patients. Curr Opin Clin Nutr Metab Care. 2007;10:206-209.

46. Ahmann A, Hellman R, Larsen K, Maynard G. Designing and implementing insulin infusion protocols and order sets. J Hosp Med. 2008;3(5):S42-S54.

47. Umpierrez GE, Smiley D, Zisman A, Prieto LM, Palacio A, Ceron M, et al. Randomized study of basal-bolus insulin therapy in the inpatient management of patients with type 2 diabetes (RABBIT 2 trial). Diabetes Care. 2007;30:2181-2186.

48. Society of Hospital Medicine. Glycemic control resource room. Available at: http://www.hospitalmedicine.org/ ResourceRoomRedesign/GlycemicControl.cfm. Accessed November 2007.

49. Society of Hospital Medicine. Workbook for improvement: improving glycemic control, preventing hypoglycemia, and optimizing care of the inpatient with hyperglycemia and diabetes. Available at: http://www.hospitalmedicine.org/ 
ResourceRoomRedesign/pdf/GC_Workbook.pdf. Accessed November 2007.

50. Garber AJ, Moghissi ES, Bransome ED Jr, et al. American College of Endocrinology position statement on inpatient diabetes and metabolic control. Endocr Pract. 2004;10:77-82.

51. Maynard G, Lee JH, Phillips G, Fink MA, Renvall M. Improved inpatient use of basal insulin, reduced hypoglycemia, and improved glycemic control: effect of structured subcutaneous insulin orders and an insulin management algorithm. J Hosp Med. 2008. In press.

52. American Association of Clinical Endocrinologists Inpatient Glycemic Control Resource Center. 2007. Available at: http:// resources.aace.com/index.asp. Accessed December 2007.

53. American Heart Association. Get With the Guidelines. Available at: http://www.americanheart.org/getwiththeguidelines. Accessed November 2007.

54. Joint Commission. Disease Specific-Care Certification. Available at:http://www.jointcommission.org/Certification Programs. Accessed November 2007.

55. The Joint Commission Disease-Specific Certification Program. Range JE. Oncology issues. July/August 2007:40-41.

56. Anonymous. The Diabetes Control and Complications Trial Research Group (DCCT). The effect of intensive treatment of diabetes on the development and progression of longterm complications in insulin-dependent diabetes mellitus. N Engl J Med. 1993;329:977-986.

57. Intensive blood-glucose control with sulphonylureas or insulin compared with conventional treatment and risk of complications in patients with type, 2 diabetes (UKPDS 33). UK Prospective Diabetes Study (UKPDS) Group. Lancet. 1998;352:837-853.

58. Gaede P, Vedel P, Parving H-H, Pedersen OU. Intensified multifactorial intervention in patients with type 2 diabetes mellitus and microalbuminuria: the Steno type 2 randomised study. Lancet. 1999;353:617-622.

59. Greci LS, Kailasam M, Malkani S, et al. Utility of HbAlc levels for diabetes case finding in hospitalized patients with hyperglycemia. Diabetes Care. 2003;26:1064-1068.

60. Baldwin D, Villanueva G, McNutt R, Bhatnagar S. Eliminating inpatient sliding-scale insulin: a reeducation project with medical house staff. Diabetes Care. 2005;28:1008-1011.

61. Warshaw HS, Bolderman KM. Advanced carbohydrate counting. In: Practical Carbohydrate Counting: A How-toTeach Guide for Health Professionals. Alexandria, VA: American Diabetes Association;2001:26-28.

62. Pastors JG, Warshaw H, Daly A, Franz M, Kulkarni K. The evidence for the effectiveness of medical nutrition therapy in diabetes management. Diabetes Care. 2002;25:608-613.

63. Boucher JL, Swift CS, Franz MJ, et al. Inpatient management of diabetes and hyperglycemia: implications for nutrition practice and the food and nutrition professional. J Am Diet Assoc. 2007;107:105-111.

64. Braithwaite SS. The transition from insulin infusions to long-term diabetes therapy: the argument for insulin analogs. Semin Thorac Cardiovasc Surg. 2006;18:366-378.

65. O’Malley. Transitions paper. J Hosp Med. 2008.

66. Schnipper JL, Barsky EE, Shaykevich S, Fitzmaurice G, Pendergrass ML. Inpatient management of diabetes and hyperglycemia among general medicine patients at a large teaching hospital. J Hosp Med. 2006;1:145-150.

67. Phillips LS, Branch WT, Cook CB, et al. Clinical inertia. Ann Intern Med. 2001;135:825-834.
68. Cook CB, Castro JC, Schmidt RE, et al. Diabetes care in hospitalized noncritically ill patients: more evidence for clinical inertia and negative therapeutic momentum. J Hosp Med. 2007;2:203-211.

69. University HealthSystem Consortium. Glycemic control 2005 findings and conclusions. Presented at: Glycemic Control 2005 Knowledge Transfer Meeting; 2005 August 19, 2005; Chicago, IL.

70. Umpierrez G, Maynard G. Glycemic chaos (not glycemic control) still the rule for inpatient care: how do we stop the insanity? J Hosp Med. 2006;1:141-144.

71. Golightly LK, Jones MA, Hamamura DH, Stolpman NM, McDermott MT. Management of diabetes mellitus in hospitalized patients: efficiency and effectiveness of slidingscale insulin therapy. Pharmacotherapy. 2006;26:1421-1432.

72. Varghese P, Gleason V, Sorokin R, Senholzi C, Jabbour S, Gottlieb JE. Hypoglycemia in hospitalized patients treated with antihyperglycemic agents. J Hosp Med. 2007;2: 234-240.

73. Fischer KF, Lees JA, Newman JH. Hypoglycemia in hospitalized patients. N Engl J Med. 1986;315:1245-1250.

74. Vriesendorp TM, van Santen S, DeVries JH, et al. Predisposing factors for hypoglycemia in the intensive care unit. Crit Care Med. 2006;34:96-101.

75. Svensson AM, McGuire DK, Abrahamsson P, Dellborg M. Association between hyper- and hypoglycaemia and 2 year all-cause mortality risk in diabetic patients with acute coronary events. Eur Heart J. 2005;26:1255-1261.

76. Pinto DS, Skolnick AH, Kirtane AJ, et al. U-shaped relationship of blood glucose with adverse outcomes among patients with ST-segment elevation myocardial infarction. J Am Coll Cardiol. 2005;46:178-180.

77. Eshaghian S, Horwich TB, Fonarow GC. An unexpected inverse relationship between HbAlc levels and mortality in patients with diabetes and advanced systolic heart failure. Am Heart J. 2006;151:91.

78. Kosiborod M, Inzucchi SE, Krumholz HM, et al. Glucometrics in patients hospitalized with acute myocardial infarction: defining the optimal outcomes-based measure of risk. Circulation. 2008;117:1018-1027.

79. Cryer PE, Davis SN, Shamoon H. Hypoglycemia in diabetes. Diabetes Care. 2003;26:1902-1912.

80. Bhatia A, Cadman B, Mackenzie I. Hypoglycemia and cardiac arrest in a critically ill patient on strict glycemic control. Anesth Analg. 2006;102:549-551.

81. Scalea TM, Bochicchio GV, Bochicchio KM, Johnson SB, Joshi M, Pyle A. Tight glycemic control in critically injured trauma patients. Ann Surg. 2007;246:605-610; discussion $10-12$.

82. Krinsley JS, Grover A. Severe hypoglycemia in critically ill patients: risk factors and outcomes. Crit Care Med. 2007;35: 2262-2267.

83. Garg R, Bhutani H, Jarry A, Pendergrass M. Provider response to insulin-induced hypoglycemia in hospitalized patients. J Hosp Med. 2007;2:258-260.

84. Newton CA, Young S. Financial implications of glycemic control: results of an inpatient diabetes management program. Endocr Pract. 2006;12(Suppl 3):43-48.

85. Levetan CS, Salas JR, Wilets IF, Zumoff B. Impact of endocrine and diabetes team consultation on hospital length of stay for patients with diabetes. Am J Med. 1995; 99:22-28. 